\title{
The Early Induction of Suppressor of Cytokine Signaling 1 and the Downregulation of Toll-like Receptors 7 and 9 Induce Tolerance in Costimulated Macrophages
}

\author{
Hyo-Ji Lee ${ }^{1,2}$, Keun-Cheol Kim², Jeong A Han ${ }^{3}$, Sun Shim Choi', and Yu-Jin Jung ${ }^{1,2 *}$
}

Toll-like receptors (TLR) 7 and 9 transduce a cellular signal through the MyD88-dependent pathway and induce the production of inflammatory mediators against microbial nucleotide components. The repeated stimulation of TLR4 leads to endotoxin tolerance, but the molecular mechanisms of tolerance induced through the costimulation of individual TLR has not yet been established, although endosomal TLRs share signaling pathways with TLR4. In the present study, mouse macrophages were simultaneously stimulated with the TLR7 agonist, gardiquimod (GDQ), and the TLR9 agonist, CpG ODN 1826, to examine the mechanism and effector functions of macrophage tolerance. Compared with individual stimulation, the costimulation of both TLRs reduced the secretion of TNF- $\alpha$ and IL-6 through the delayed activation of the NF-KB pathway; notably, IL-10 remained unchanged in costimulated macrophages. This tolerance reflected the early induction of suppressor of cytokine signaling-1 (SOCS-1), according to the detection of elevated $T N F_{-\alpha}$ secretion and restored NF$\kappa B$ signaling in response to the siRNA-mediated abrogation of SOCS-1 signaling. In addition, the restimulation of each TLRs using the same ligand significantly reduced the expression of both TLRs in endosomes. These findings revealed that the costimulation of TLR7 and TLR9 induced macrophage tolerance via SOCS-1, and the restimulation of each receptor or both TLR7 and TLR9 downregulated $T L R$ expression through a negative feedback mechanisms that protects the host from excessive inflammatory responses. Moreover, the insufficient and impaired immune response in chronic viral infection might also reflect the repeated and simultaneous stimulation of those endosomal TLRs.

\footnotetext{
${ }^{1}$ BIT Medical Convergence Graduate Program, ${ }^{2}$ Department of Biological Sciences, ${ }^{3}$ Department of Biochemistry and Molecular Biology, School of Medicine, ${ }^{4}$ Department of Medical Biotechnology, Kangwon National University, Chuncheon 200-701, Korea

*Correspondence: yjjung@kangwon.ac.kr
}

Received 23 May, 2014; revised 29 September, 2014; accepted 21 October, 2014; published online 15 December, 2014

Keywords: inflammation, macrophage, NF-kB, SOCS-1, TLRs, tolerance

\section{INTRODUCTION}

The host innate immune system is a first line defense mechanism against bacterial, viral and fungal infection (Akira, 2009). The activation of the innate immune response rapidly regulates the replication of microorganisms and induces the development of adaptive immunity. Antigen-presenting cells (APCs), such as macrophages, dendritic cells (DCs) and B cells, express various pattern recognition receptors (PRRs) and recognize pathogen derived pathogen-associated molecular patterns (PAMPs), including lipids, proteins, lipoproteins and nucleic acids (Labonte et al., 2014; Pluddemann et al., 2011). TLR is one of the best studied PRR families, and these proteins recognize various microorganism-derived nucleotides, such as dsRNA (TLR3), ssRNA (TLR7) and bacterial CpG DNA (TLR9). After recognition of specific ligands, most TLRs, except TLR3, induce the recruitment of adaptor proteins, such as MyD88, IRAK and TRAF6, and increase the expression of inflammatory cytokines, type I interferon (IFN), chemokines and costimulatory molecules through the activation of NF-kB and MAPK pathways (Chang, 2010).

Although the inflammatory response regulates the growth of microorganisms to protect host cells, immoderate cytokine production damages the host cells (Cross et al., 1995; Danner et al., 2009; Lee and Hwang, 2006). Recent studies have suggested that repeated exposure to endotoxin (such as lipopolysaccharide; LPS) induces an "endotoxin tolerance", which does not respond to endotoxin (Fujita, 2009; Koons et al., 2008). Endotoxin tolerance is a negative feedback mechanism to protect the host against endotoxin shock and the negative regulation of TLR signaling through proteins, such as IRAK-M, SOCS1 and A20 (Hassan et al., 2009; Liu et al., 2008a; Xiong and Medvedev, 2011). Therefore, innate immunity is tightly controlled through the signaling of negative regulators that inhibit excessive cytokine production. However, the tolerance induced through the repetitive stimulation of other TLR ligands or simultaneous stimulation of different TLR ligands remains largely unknown.

In the present study, we examined whether costimulation with agonists for TLR7 and TLR9 or subsequent stimulation with the same agonists induced tolerance in mouse macrophages. The costimulation or restimulation of mouse macrophages with gardiquimod (GDQ, TLR7 agonist) and CpG oligodeoxynucleotide 1826 (ODN1826, TLR9 agonist) reduced the levels of proinflammatory cytokines TNF- $\alpha$ and IL-6, but the 
levels of anti-inflammatory cytokine IL-10 remained unchanged. Costimulation with agonists for TLR7 and TLR9 also delayed the activation of NF- $\mathrm{KB}$ signaling and increased the levels of SOCS-1. In addition, the expression of each TLR was significantly decreased after restimulation, while the TLR expression was rapidly recovered when the cells were stimulated with a single agonist alone. Therefore, the costimulation of TLR7 and TLR9 induced macrophage tolerance via the delayed transduction of NF$\kappa \mathrm{B}$ signaling and SOCS-1 activity, whereas restimulation downregulated the expression of TLR in mouse macrophages.

\section{MATERIALS AND METHODS}

\section{Reagents}

Gardiquimod (GDQ) (TLR7 agonist) and lipopolysaccharide (LPS) (TLR4 agonist) were purchased from InvivoGen (USA). The TLR9 agonist CpG oligodeoxynucleotide 1826 (ODN1826), which is phosphorothioated backbone-modified oligodeoxynucleotide, was purchased from GenoTech (Korea). All antibodies were purchased from Santa Cruz Biotechnology (USA) except anti-phospho-IKB, anti-phospho-MEK, anti-MEK, anti-phosphoErk1/2 and anti-Erk1/2 (Cell Signaling Technologies, USA).

\section{Cell culture and BMDM isolation}

The mouse macrophage cell line, RAW264.7 was purchased from the American Type Culture Collection (ATCC) and maintained in RPMI1640 (Lonza, USA) culture medium containing $10 \%$ heat-inactivated fetal bovine serum (FBS; Lonza, USA) and penicillin (10,000 U/L) (Gibco-BRL, USA) at $37^{\circ} \mathrm{C}, 5 \% \mathrm{CO}_{2}$. Mouse bone marrow-derived macrophages (BMDMs) were isolated from 8-week-old wild-type C57BL/6 and BALB/c male mice (Nara Bio., Korea). Briefly, BMDMs were obtained after culturing bone marrow-derived mononuclear precursor cells isolated from the femurs and tibiae in RPMI1640 medium supplemented with $10 \%$ heat-inactivated fetal bovine serum, penicillin (10,000 U/L) and 20\% L-929 cell conditioned medium at $37^{\circ} \mathrm{C}$ and $5 \% \mathrm{CO}_{2}$ for 3 days.

\section{Cell transfection}

The siRNA target sequence for mouse SOCS-1 was purchased from Santa Cruz Biotechnology (USA). The cells were transfected with 60 pmol siSOCS-1 using the Lipofectamine reagent (Invitrogen, USA) in serum-free medium according to the manufacturer's instructions. The cells were incubated at $37^{\circ} \mathrm{C}$ and $5 \% \mathrm{CO}_{2}$ for $6 \mathrm{~h}$ and recovered for an additional $6 \mathrm{~h}$ before treatment with TLR agonists.

\section{Enzyme-linked immunosorbent assay (ELISA)}

The concentration of mouse TNF- $\alpha$, IL- 6 and IL-10 in the cell culture media was determined using an enzyme-linked immunosorbent assay (ELISA). RAW264.7 cells and BMDMs $\left(1 \times 10^{5}\right.$ cells/well) were seeded onto a 24 -well plate and stimulated with either agonists of TLR7 and TLR9 or both at $37^{\circ} \mathrm{C}$ and $5 \% \mathrm{CO}_{2}$. After overnight incubation, the cells were washed with PBS (phosphate-buffered saline; Gibco BRL Life Technologies, USA) and incubated overnight in RPMl1640 medium. Subsequently, the cells were restimulated with TLR agonists for 1 $\mathrm{h}$ under the same conditions. Cell-free supernatants were obtained from siRNA-transfected cells. The murine TNF- $\alpha, \mathrm{IL}-6$ and IL-10 ELISA development kit was purchased from Peprotech (USA) and the mouse IL-1 $\beta$ and IL-1Ra Duoset $\AA$ ELISA development system was purchased from R\&D System, Inc. (USA). The cytokine levels in cell-free supernatants were determined using ELISA according to the manufacturer's instruction.

\section{Western blot analysis}

The extracted proteins were resolved on 10 to $15 \%$ SDSpolyacrylamide gels through electrophoresis, followed by transfer onto a PVDF transfer membrane (Millipore, USA). The blots were incubated with $5 \%$ skim milk in $0.1 \%$ TBS-T for $1 \mathrm{~h}$, and subsequently washed in $0.1 \%$ TBS-T (20 mM Tris, $150 \mathrm{mM}$ $\mathrm{NaCl}, \mathrm{pH} 8.0$ and 1\% Tween 20)(Kang et al., 2010). The membranes were incubated with the primary antibodies followed by washing and labeling with an HRP-conjugated secondary antibody in $0.1 \%$ TBS-T. The blot was treated with ECL (Animal Genetics Inc., Korea) and exposed to Kodak XAR film (USA) in the darkroom.

Nuclear and cytoplasmic extraction and immunoprecipitation Cells were lysed with buffer A (10 mM HEPES, $10 \mathrm{mM} \mathrm{KCl,} 0.1$ mM EGTA, 0.1 mM EDTA, 1 mM DTT, 0.3\% NP-40) containing protease inhibitor for $15 \mathrm{~min}$ and centrifuged for $5 \mathrm{~min}$ at 4,000 r.p.m. Supernatant corresponding to cytoplasm was saved and kept on ice. The pellet was resuspended with buffer $B(25 \mathrm{mM}$ HEPES, $0.5 \mathrm{mM}$ EDTA, $0.4 \mathrm{M} \mathrm{NaCl}, 1 \mathrm{mM}$ DTT) containing protease inhibitor and incubated for $30 \mathrm{~min}$ at $4^{\circ} \mathrm{C}$. Lysate was centrifugated for 10 minutes at 10,000 r.p.m and the supernatant corresponding to nuclear extract was separated for immuneprecipitation input control. For immunoprecipitation analysis, phospho-NF-кB p65 antibody or SOCS-1 antibody and protein $\mathrm{G}$ plus/protein A beads (Millipore, USA) was added in nuclear extract and incubated for overnight at $4^{\circ} \mathrm{C}$ on a rotator. Finally, lysate was washed three times with IP buffer $(50 \mathrm{mM}$ Tris- $\mathrm{HCl}, \mathrm{pH} 7.5,250 \mathrm{mM} \mathrm{NaCl}, 0.5 \% \mathrm{NP}-40$ ), and denaturated with SDS at $95^{\circ} \mathrm{C}$ for $5 \mathrm{~min}$.

\section{Total RNA isolation and RT-PCR}

The RNA extraction was performed using an RNeasy mini kit (Qiagen, USA) according to the manufacturer's instruction. Briefly, $2 \mu \mathrm{g}$ of total RNA was reverse transcribed using M-MLV RT (Enzynomics, Korea) in a reaction including RNase inhibitors and a random hexamer (Takara, Japan) according to the manufacturer's instructions. The PCR reaction was performed with forward and reverse primers and $0.5 \mu$ of Taq polymerase (Takara, Japan). The nucleotide sequences of the RT-PCR primers and the sizes of amplified products are described in Supplementary Table S1. The amplified products were separated on $1.5 \%$ agarose gel and visualized after staining with ethidium bromide (Sigma-Aldrich, USA). The expression of the housekeeping genes, glyceraldehyde-3-phosphate dehydrogenase (GAPDH) and $\beta$-actin was determined as an internal control.

\section{Luciferase assay}

RAW264.7 cells were transiently transfected with a NF-кB reporter construct (Promega, USA) using lipofectamine 2000. The cells were stimulated with either agonists for TLR7 and TLR9 or both. Subsequently, the cells were washed with PBS and incubated overnight in RPMI 1640 medium at $37^{\circ} \mathrm{C}$ and $5 \%$ $\mathrm{CO}_{2}$. And then, the cells were restimulated with TLR agonists for $1 \mathrm{~h}$ under the same conditions and lysed in $100 \mu$ lof luciferase cell culture lysis reagent (Promega, USA). The NF-kB promoter activity was measured using the Dual-Luciferase Reporter Assay System (Promega, USA) and the results were obtained using luminometer according to the manufacturer's specifications. 
TLR9 or both for 4 or $16 \mathrm{~h}$, followed by fixation and permeabilization with $4 \%$ paraformaldehyde and $0.1 \%$ saponin. After washing, the cells were incubated with anti-mouse Alexa Fluor ${ }^{\circledR}$ 488-conjugated TLR7 monoclonal antibody (mAb) or antimouse Alexa Fluor ${ }^{\circledR}$ 488-conjugated TLR9 mAb (IMGENEX, USA) for $30 \mathrm{~min}$ at $4^{\circ} \mathrm{C}$ in the dark. Subsequently, the cells were washed twice with cold PBS, supplemented with $2 \%$ FBS. The absorbance was read on a BD-FACSCalibur instrument (BD-Biosciences, USA) and flow cytometric analysis was performed with Cell Quest (BD-Biosciences) software.

\section{Statistical analysis}

The data were obtained from independent experiments. The levels of significance for comparison between samples were determined with unpaired Student's t-tests using GraphPad Prism 5 software (GraphPad Software, USA). Statistical significance was indicated as ${ }^{*} \mathrm{P}<0.05,{ }^{* \star} \mathrm{P}<0.01,{ }^{* *} \mathrm{P}<0.001$ and n.s., not significant $(P<0.05)$.

\section{RESULTS}

TLR stimulation induces the innate immune response through the increased secretion of proinflammatory cytokines, such as TNF- $\alpha$, IL-1 $\beta$ and IL-6 (Akira, 2009). To determine whether the excessive stimulation of both TLR7 and TLR9 induces hyperresponsiveness or hyporesponsiveness in mouse macrophages, proinflmmatory and anti-inflammatory cytokines were measured using an ELSIA assay. The production of TNF- $\alpha$ was decreased when RAW264.7 cells were simultaneously stimulated with agonists for TLR7 and TLR9 compared with cells stimulated agonists for either TLR7 or TLR9 alone (Fig. 1A). Decreased TNF- $\alpha$ expression was observed in both BALB/C and C57BL/6 BMDMs (Fig. 1A). Moreover, the restimulation of TLRs decreased TNF- $\alpha$ secretion, when macrophages were stimulated with agonists for either TLR7 or TLR9 or both overnight, followed by treatment with the same agonists for $1 \mathrm{~h}$ (Fig. 1B). IL-6 levels were significantly reduced in both costimulated and restimulated groups (Fig. 1C). However, the secretion of IL-10 and IL-1 $\beta$ remained unchanged (Fig. 1D and Supplementary Fig. S1). These results showed that costimulation and restimulation with specific agonists for TLR7 and TLR9 induced tolerance through a reduction in the levels of proinflammatory cytokines in mouse macrophages.

The association of TLRs with specific ligands stimulates the recruitment of TLR signaling mediators, such as IRAK4, IRKA1, TRAF6 and the IKK family, through the MyD88 pathway, resulting in the subsequent activation of NF-KB (Kawai and Akira, 2007). The MyD88 pathway also activates the MAPK pathway. To further examine the reduction of proinflammatory cytokine expression in macrophages costimulated with both agonists for TLR7 and TLR9, the molecules involved in the NF-кB pathway and the MAPK pathway were determined using western blot analysis. IRAK-1, which transmits a signal to the IKK complex via degradation through auto-phosphorylation, was degraded at $1 \mathrm{~h}$ after stimulation with a single agonist, but the expression of this molecule was sustained at $3 \mathrm{~h}$ after costimulation of both TLRs (Fig. 2A). The rapid degradation of $I \kappa B \alpha$ results in the translocation of NF- $\kappa B$ to the nucleus; less $l_{\kappa} \mathrm{B} \alpha$ degradation was detected when cells were stimulated with both agonists for TLR7 and TLR9 compared with cells stimulated with a single agonist (Fig. 2A). In addition, the delayed translocation of NF$\kappa B$ p65 to nuclear was observed when RAW264.7 cells were simultaneously stimulated with agonists for TLR7 and TLR9 (Fig. 2B). However, the expression of phosphorylated MEK, ERK and
A
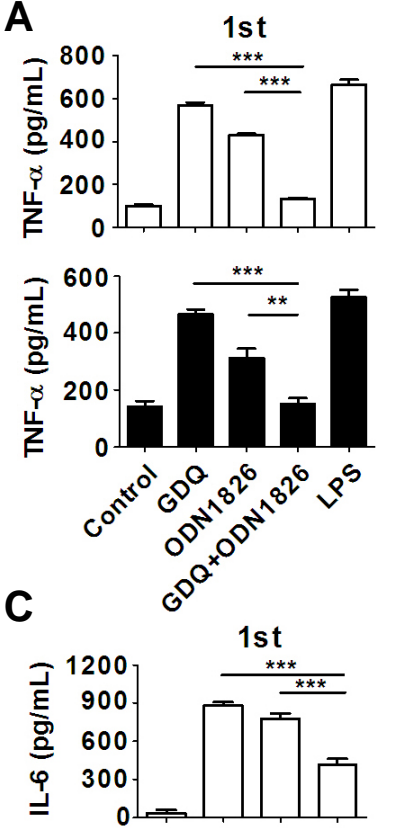

D

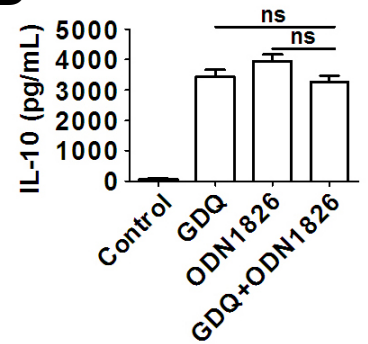

$B$
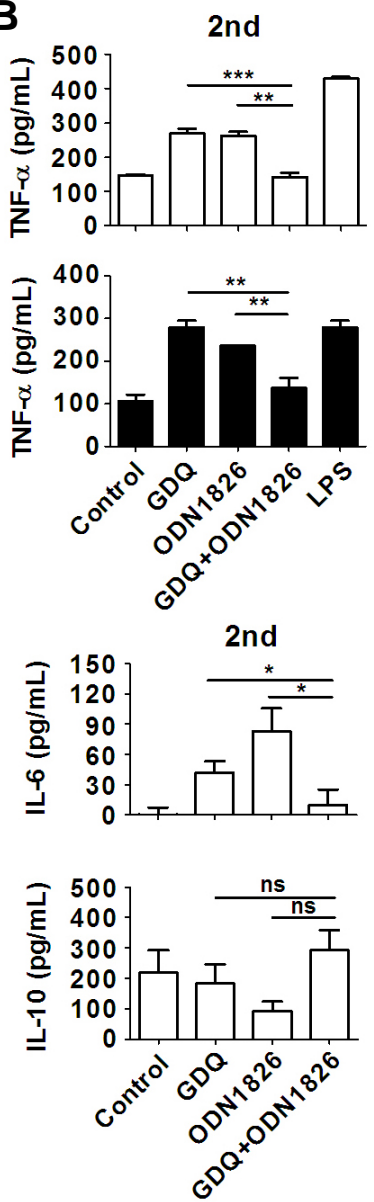

Fig. 1. Costimulation and/or restimulation of TLR7 and TLR9 decreased the levels of proinflammatory cytokine production in mouse macrophages. (A) Mouse macrophages were stimulated with either an agonist for TLR7 (GDQ) or TLR9 (ODN 1826) or both (GDQ + ODN1826) overnight (1st), and the production of TNF- $\alpha$ in the supernatant was measured using ELISA. LPS stimulation was introduced as a positive control. $\square$ Raw264.7 macrophage cell line, and - BMDMs from Balb/c mice. (B) After the first stimulation, the cells were washed with PBS and incubated with culture media overnight. Subsequently, the cells were restimulated with TLR agonists for $1 \mathrm{~h}$ (2nd), and the production of TNF- $\alpha$ was measured using ELISA. The levels of IL-6 (C) and IL-10 (D) were measured in RAW264.7 cells under the same conditions. Significant differences were indicated as ${ }^{*} P<0.05,{ }^{* *} P<0.01,{ }^{* * *} P<0.001$, and $n . s$., not significant $(P<0.05)$.

p38 remained unchanged between single and costimulated samples.

To further confirm the reduction in NF- $\mathrm{KB}$ signaling through costimulation or restimulation with agonists for TLR7 and TLR9, the promoter activity of NF- $\mathrm{KB}$ was assessed using luciferase assays. As shown in Fig. 2C, NF-кB promoter activity was significantly decreased when macrophages were simultaneously stimulated or restimulated with both agonists compared with cells stimulated with a single agonist (Fig. 2C).

Negative regulators of TLR signaling, such as A20, IRAK-M, SOCS- 1 and SOCS-3, reduce or terminate the innate immune response (Hubbard and Moore, 2010; Lang and Mansell, 2007). 
A
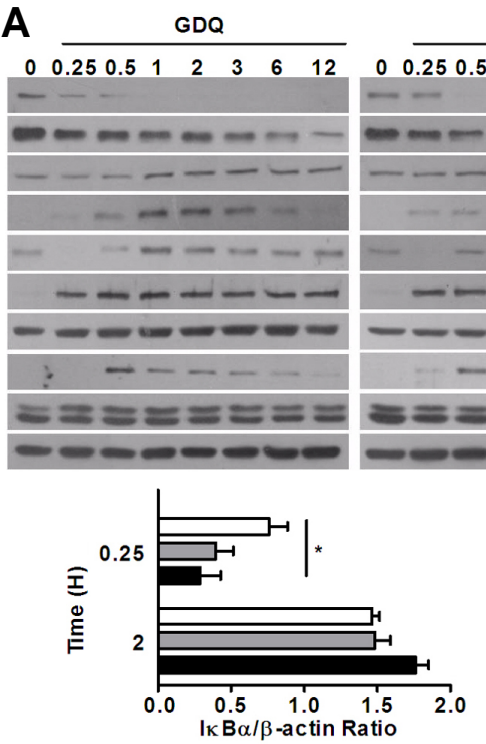

ODN1826

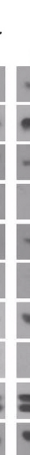

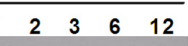

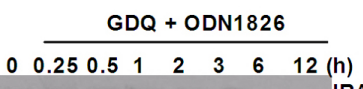

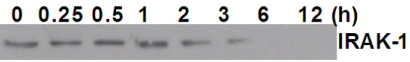
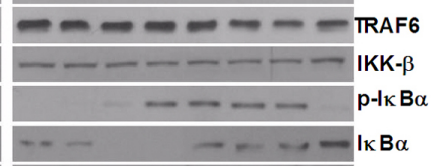

$---b-M-M E K$

$-----2-1 \mathrm{MEK}$ $\mathrm{p}-\mathrm{Erk} 1 / 2$

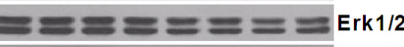
$-2-2-a-a c t i n$

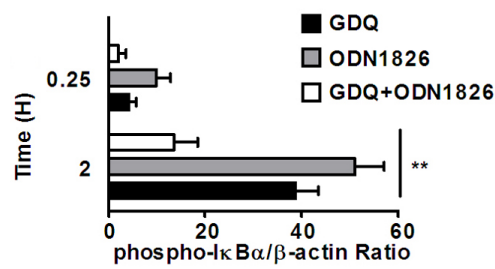

B

ODN1826

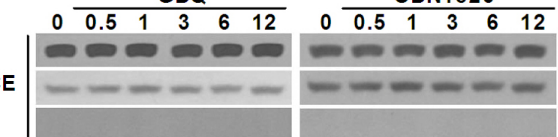

$0 \frac{\mathrm{GDQ}+O D N 1826}{0.5133612(\mathrm{~h})}$
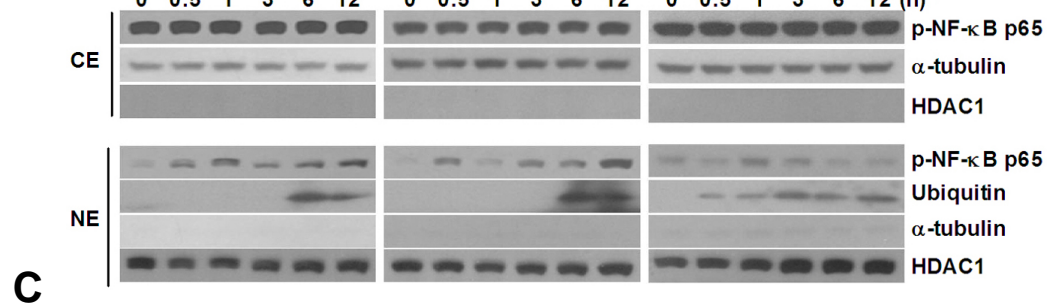

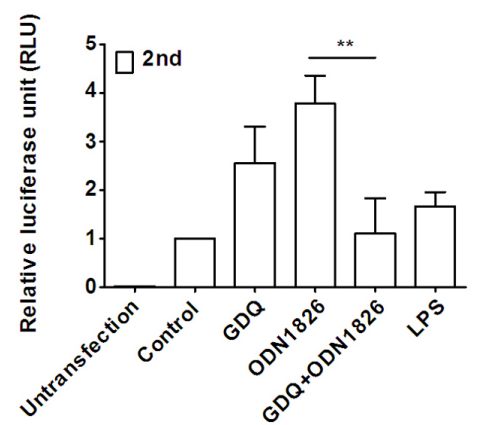

Fig. 2. Delayed activation of NF-kB signaling in cells simultaneously stimulated with both agonists for TLR7 and TLR9. (A) RAW264.7 cells were stimulated with either an agonist for TLR7 or TLR9 or both. The cell lysates were extracted, and the changes in expression levels of TLR signaling downstream molecules were assessed using Western blot analysis. Bar graphs indicate the results of the relative densitometry compared with $\beta$-actin protein. (B) Nuclear translocation of p65 was identified within cytoplasmic (CE) and nuclear fractions (NE) by Western blotting against p65. (C) RAW264.7 cells were transfected with the NF-kB-luc reporter gene. The transfected cells were treated with either an agonist for TLR7 and TLR9 or both for $4 \mathrm{~h}$ (1st), followed by washing with PBS and incubation in culture media for $12 \mathrm{~h}$. Subsequently, the cells were restimulated with TLR agonists for $1 \mathrm{~h}(2 \mathrm{nd})$, and the NF-KB promoter activity was measured through luciferase assay. Significant differences were indicated as ${ }^{* *} \mathrm{P}<0.01,{ }^{* *} \mathrm{P}<$ 0.001 , and n.s., not significant $(\mathrm{P}<$ $0.05)$.
Thus, involvement of negative regulators of TLR signaling in the induction of tolerance in mouse macrophages costimulated with both TLR7 and TLR9 was assessed. The expression of SOCS-1 was significantly increased at $30 \mathrm{~min}$ to $1 \mathrm{~h}$ after the costimulation of RAW264.7 cells; notably, there was no change in the expression levels of IRAK-M or SOCS-3 (Figs. 3A and 3B). After stimulation with agonists for either TLR7 or TLR9 or both overnight, the level of TNF- $\alpha$ secretion was significantly increased in the cells with transiently abolished SOCS -1 mRNA expression compared with cells transfected with scrambled RNA (Fig. 3C). The inhibition of SOCS-1 did not significantly affect the levels of TNF- $\alpha$ in siSOCS-1-transfected RAW264.7 cells restimulated with agonists for either TLR7 and TLR9 or both (Fig. 3C).

To identify whether the delayed NF-кB activity is due to the degradation of ubiquitinated p65 subunit, expression of molecules involved in NF-kB signaling pathway were measured after knockdown of SOCS1 using siRNA in RAW264.7 cells.
The rapid degradation and phosphorylation of $I_{\kappa} \mathrm{B} \alpha$ was observed in siSOCS-1-knockdown macrophages stimulated with agonists for either TLR7 or TLR9 or both (Fig. 4A). Furthermore, to investigate whether endogenous SOCS-1 and NF-kB p65 interact in nucleus, phosphor-NF- $\mathrm{kB}$ p65 or SOCS-1 was immuno- precipitated from nuclear extracts of macrophage stimulated with single or both agonists. Endogenous p65 and SOCS-1 were coimmunoprecipitated and costimulation induced a stronger p65 ubiquitination in mouse macrophages compared with single stimulation (Fig. 4B).

A previous study has shown that the expression of TLRs was reduced through the stimulation of TLRs with specific agonists (Shuto et al., 2005). To verify whether the observed macrophage tolerance reflected the inadequate expression of TLRs in cells restimulated with either or both agonists, the expression of TLR7 and TLR9 was measured at the transcriptional and translational levels. The results showed that the expression of TLR7 


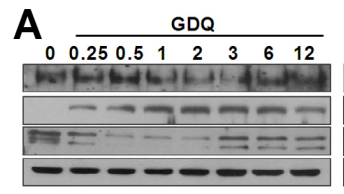

\section{B}
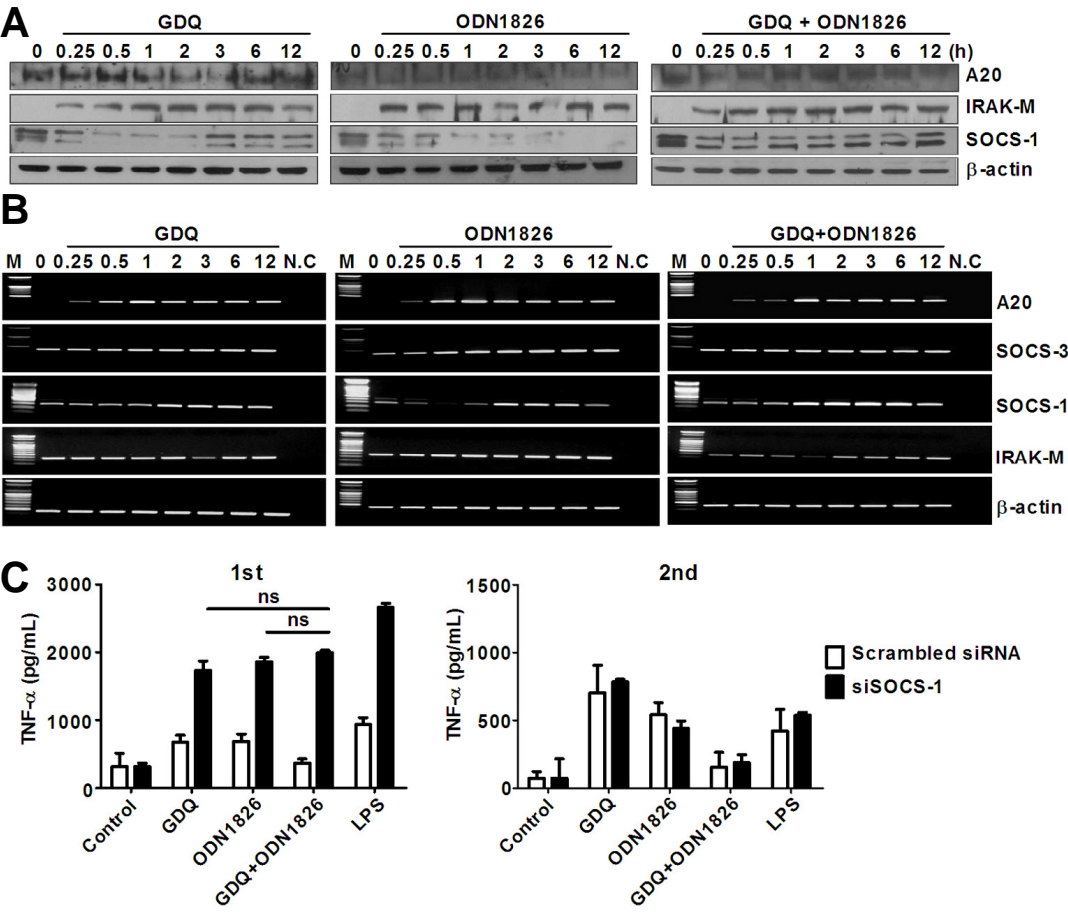

$\boldsymbol{A}$

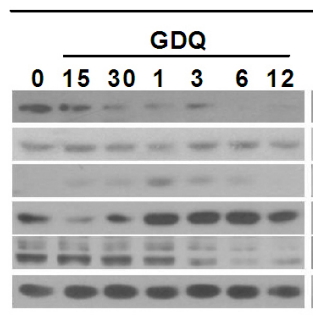

siscr

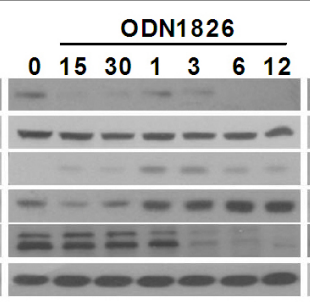

GDQ+ODN1826

$\begin{array}{lllllll}0 & 15 & 30 & 1 & 3 & 6 & 12\end{array}$

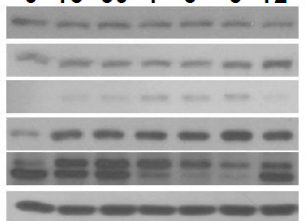

IRAK-1

IKK- $\beta$

$p-I \kappa B \alpha$

I $B \alpha$

socs - 1

sisocs-1

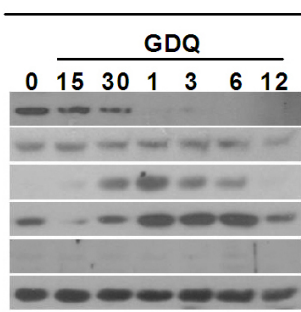

ODN1826

$\begin{array}{lllllll}0 & 15 & 30 & 1 & 3 & 6 & 12\end{array}$

GDQ+ODN1826

$\begin{array}{llllllll}0 & 15 & 30 & 1 & 3 & 6 & 12 & \text { (h) }\end{array}$
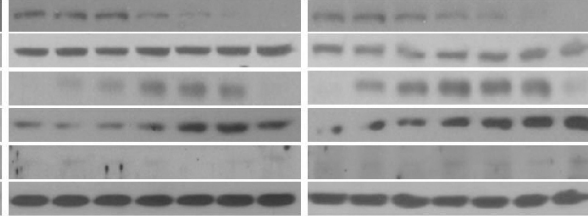

IRAK-1

IKK- $\beta$

$p-I \kappa B \alpha$

I $B \alpha$

SOCS -1

$\alpha$-tubulin

B
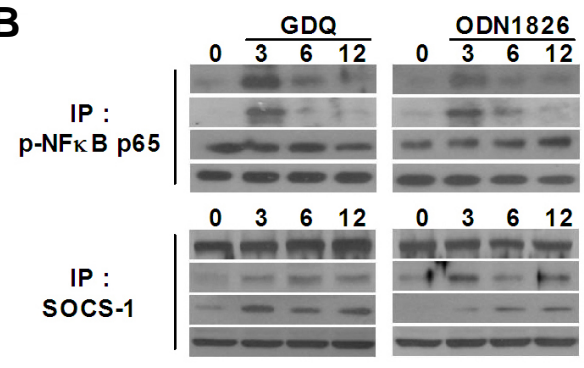

GDQ+

ODN1826

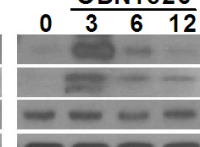

SOCS-1

Ubiquitin

p-NFK B p65
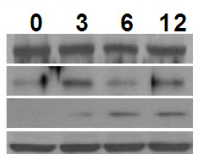

$\begin{array}{llll}0 & 3 & 6 & 12\end{array}$

HDAC1

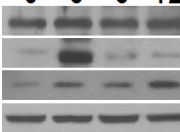

socs-1

Ubiquitin

p-NFK B p65

- HDAC1

$\begin{array}{llll}0 & 3 & 6 & 12\end{array}$

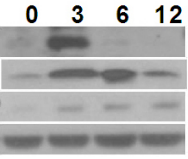

socs-1

Ubiquitin

p-NFKB p65

HDAC1
Fig. 3. Upregulation of SOCS1 after TLR7 and TLR9 costimulation. (A) RAW264.7 cells were stimulated with either an agonist for TLR7 or TLR9 or both for the indicated time. The protein expression in the total lysates was analyzed by Western blotting using antibodies against A20, IRAK-M, SOCS-1 and $\beta$-actin. (B) The mRNA expression levels of A20, SOCS-3, SOCS-1, IRAK-M and $\beta$-actin were determined through RT-PCR. (C) RAW264.7 cells were transfected with siRNA targeting SOCS-1 mRNA or scrambled siRNA and stimulated with either an agonist for TLR7 or TLR9 or both overnight (1st). After stimulation, the cells were washed with PBS and incubated with culture media overnight. Subsequently, the cells were restimulated with TLR agonists for $1 \mathrm{~h}(2 \mathrm{nd})$ and the production of TNF- $\alpha$ was measured through ELISA.
Fig. 4. Recovered NF- $\mathrm{kB}$ signaling in the costimulated cells when SOCS-1 was abolished $(A)$ RAW264.7 cells were transfected with siRNA targeting SOCS-1 mRNA or scrambled siRNA and stimulated with either an agonist for TLR7 or TLR9 or both for indicated time points. The whole cell lysates were extracted, and Western blot analysis showed the cellular levels of IRAK- 1 , IKK- $\beta$, IкB $\alpha$ and SOCS-1. The $\alpha$-tubulin was used as a loading control. (B) RAW264.7 cells were stimulated with each agonist for TLR7 and TLR9 or both for indicated time points, and then nuclear extracts were immunoprecipitated with anti-phospho-NF-кB p65 or anti-SOCS-1, followed by Western blot analysis. 
A
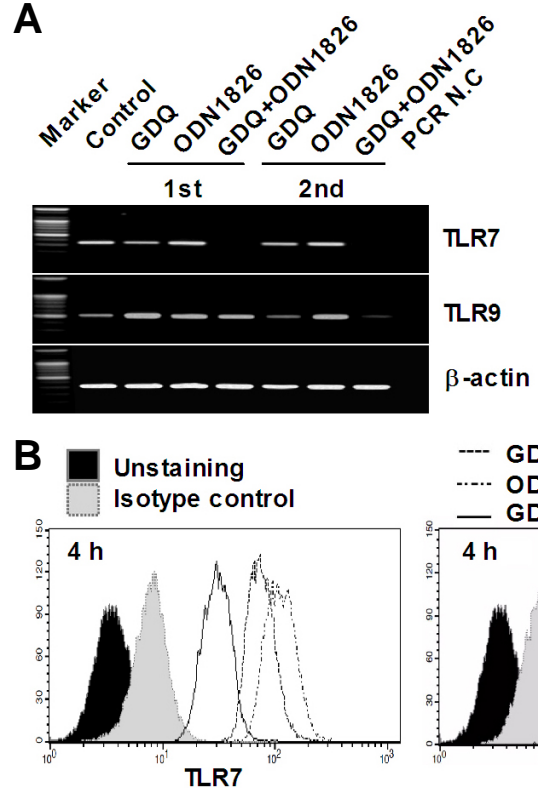

--- GDQ

ODN1826
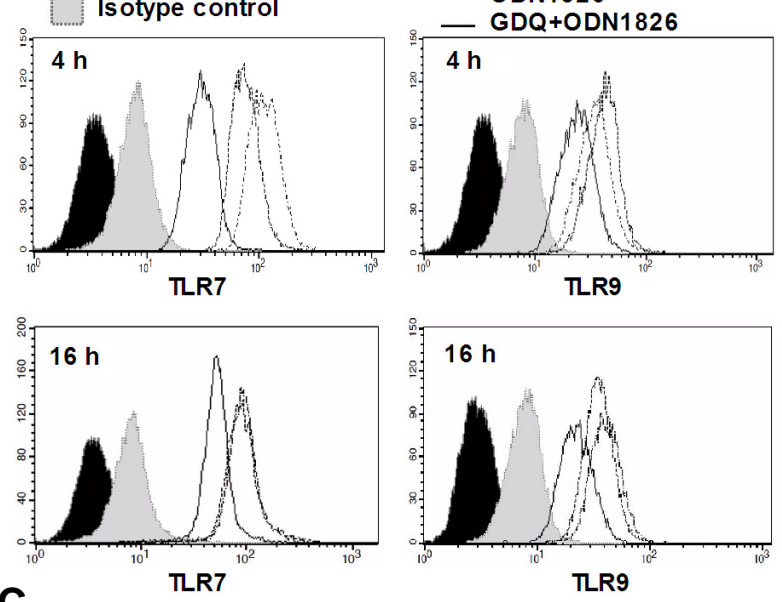

C
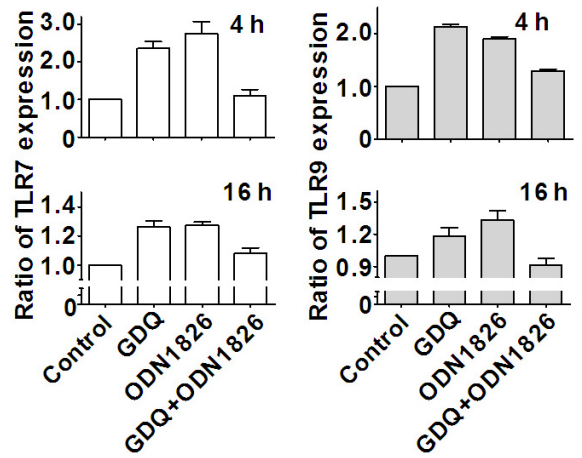

Fig. 5. The costimulation and/or restimulation of TLR7 and TLR9 decrease the expression of TLR7 and TLR9. (A) RAW264.7 cells were stimulated with either an agonist for TLR7 or TLR9 or both overnight (1st), subsequently the cells were washed with PBS and incubated with culture media overnight. After incubation, the cells were restimulated with TLR agonists for $1 \mathrm{~h}$ (2nd) and the expression of TLR7 and TLR9 was measured using RT-PCR. (B) The cells were incubated with either an agonist for TLR7 and TLR9 or both for 4 or $16 \mathrm{~h}$, and the expression of endosomal TLR7 and TLR9 was measured using flow cytometry. The data are presented as the mean fluorescence intensity (MFI) (C).

or TLR9 mRNA was less detectable when RAW264.7 cells were restimulated with each agonist alone (Fig. 5A). The expression of both TLR7 and TLR9 was significantly decreased when the cells were simultaneously restimulated with both agonists. The decreased expression of TLR7 and TLR9 proteins was detected when both TLRs were stained with fluorescence dye-conjugated antibodies and measured using flow cytometry. The expression of TLR7 and TLR9 was restored at $4 \mathrm{~h}$ after stimulation with each agonist; in contrast, both TLRs expression was not restored until $16 \mathrm{~h}$ after costimulation with both agonists (Figs. 5B and $5 \mathrm{C}$ ). These results suggested that single TLR stimulation diminished TLR expression for several hours, while costimulation reduced the expression of both TLRs for at least $16 \mathrm{~h}$.

\section{DISCUSSION}

The results obtained in the present study demonstrated that the simultaneous stimulation and restimulation of TLR7 and TLR9 with specific agonists induced tolerance in mouse macrophages, thereby reducing the production of proinflammatory cytokines TNF- $\alpha$ and IL-6. The simultaneous stimulation-mediated tolerance impaired NF- $\mathrm{KB}$ activation through the delayed degradation of IRAK-1 and IKB, which reduced the secretion of these cytokines.

Recent studies have shown that the expression of negative regulators, such as SOCS-1 (Liu et al., 2008a), IRAK-M (Harada et al., 2006; Liu et al., 2008b; Xiong and Medvedev, 2011; Zacharioudaki et al., 2009), SH2-containing inositol-5'phosphatase-1 (SHIP-1) (Xiong and Medvedev, 2011; Xiong et al., 2011) and A20 (Xiong et al., 2011), is enhanced under tolerant conditions; specifically, the involvement of IRAK-M has previously been reported in endotoxin tolerance (Liu et al., 2008b). As proposed in Fig. 3, the increased expression of SOCS-1, but not other regulators, was detected in simultaneous-stimulated mouse macrophages. In addition, the knockdown of SOCS-1 induced the increased production of TNF- $\alpha$ in the cells stimulated overnight with both agonists for TLR7 and TLR9, although SOCS-1 was not involved in the tolerance induced through the restimulated TLRs, as shown in Fig. $3 \mathrm{C}$. The inhibition of SOCS-1 was able to strongly rescue NF-KB activity in costimulated mouse macrophages, according to the accelerated degradation and phosphorylation of $I_{\kappa} \mathrm{B} \alpha$. Furthermore, following costimulation, SOCS-1 and NF- $\mathrm{KB}$ p65 subunit translocated to the nucleus, where they were interacted and induced the ubiquitination of NF-kB p65 subunit (Fig. 4). These results suggest that the delayed activation of $\mathrm{NF}-\kappa \mathrm{B}$ signaling is due to differential posttranslational modification of NF- $\mathrm{KB}$ p65 in costimulated cells. Therefore, the involvement of SOCS-1 in tolerance through simultaneous stimulation decreases TNF- $\alpha$ levels through the inhibition of NF- $\mathrm{KB}$ activation to induce the degradation of IRAK-1 and IKB (Nakagawa et al., 2002).

Taken together, these results suggest that the excessive stimulation of TLRs reduces the expression of TLRs for several hours. This finding could explain why cells costimulated with both agonists of TLR7 and TLR9 did not secrete proinflammatory cytokines during the restimulation. These endosomal TLRs play important roles in recognition and antigen processing after viral infection. Specifically, when host cells are infected with RNA viruses, such as, hepatitis $C$ virus (HCV), influenza virus and human immunodeficiency virus (HIV), host APCs could encounter viral RNA or DNA or both during viral replication. Thus, as TLR7 and TLR9 could be simultaneously or repeatedly activated, the tolerance induced through these endosomal TLRs could serve the defective innate immune mechanisms in chronic viral infections.

It has recently been reported that TLR7 expression is downregulated in the livers of HCV-infected patients (EI Tawdy and Rashed, 2012) and responses to TLR9 stimulation were 
universally decreased in all HIV-1-infected individuals (Chang et al., 2012). It has also been suggested that innate immunity is impaired after secondary bacterial infections following influenza, resulting in susceptibility to bacterial pneumonia (Chen et al., 2012).

Therefore, these findings suggest that chronic viral infection leads to immune hyporesponssiveness, and acute influenza infection induces susceptibility to secondary bacterial infections.

Note: Supplementary information is available on the Molecules and Cells website (www.molcells.org).

\section{ACKNOWLEDGMENTS}

This work was supported by the Basic Research Promotion Fund (2012R1A1A2006349) and Nuclear R\&D Program (2014030381) of the National Research Foundation of Korea (NRF). All authors declare no potential competing financial interests.

\section{REFERENCES}

Akira, S. (2009). Innate immunity to pathogens: diversity in receptors for microbial recognition. Immunol. Rev. 227, 5-8.

Chang, Z.L. (2010). Important aspects of Toll-like receptors, ligands and their signaling pathways. Inflamm. Res. 59, 791-808.

Chang, J.J., Lacas, A., Lindsay, R.J., Doyle, E.H., Axten, K.L., Pereyra, F., Rosenberg, E.S., Walker, B.D., Allen, T.M., and Altfeld, M. (2012). Differential regulation of toll-like receptor pathways in acute and chronic HIV-1 infection. Aids 26, 533-541.

Chen, W.H., Toapanta, F.R., Shirey, K.A., Zhang, L., Giannelou, A., Page, C., Frieman, M.B., Vogel, S.N., and Cross, A.S. (2012) Potential role for alternatively activated macrophages in the secondary bacterial infection during recovery from influenza. Immunol. Lett. 141, 227-234.

Cross, A., Asher, L., Seguin, M., Yuan, L., Kelly, N., Hammack, C., Sadoff, J., and Gemski, P., Jr. (1995). The importance of a lipopolysaccharide-initiated, cytokine-mediated host defense mechanism in mice against extraintestinally invasive Escherichia coli. J. Clin. Invest. 96, 676-686.

Danner, R.L., Elin, R.J., Hosseini, J.M., Wesley, R.A., Reilly, J.M., and Parillo, J.E. (2009). Endotoxemia in human septic shock. 1991. Chest 136, e30.

El Tawdy, A., and Rashed, L. (2012). Downregulation of TLR-7 receptor in hepatic and non-hepatic patients with lichen planus. Int. J. Dermatol. 51, 785-789.

Fujita, T. (2009). Molecular mechanism of endotoxin tolerance. Hepatology 50, 1322.

Harada, K., Isse, K., Sato, Y., Ozaki, S., and Nakanuma, Y. (2006) Endotoxin tolerance in human intrahepatic biliary epithelial cells is induced by upregulation of IRAK-M. Liver Int. 26, 935-942.

Hassan, F., Islam, S., Tumurkhuu, G., Dagvadorj, J., Naiki, Y., Komatsu, T., Koide, N., Yoshida, T., and Yokochi, T. (2009). Involvement of interleukin-1 receptor-associated kinase (IRAK) $\mathrm{M}$ in toll-like receptor (TLR) 7-mediated tolerance in RAW 264.7 macrophage-like cells. Cell. Immunol. 256, 99-103.

Hubbard, L.L., and Moore, B.B. (2010). IRAK-M regulation and function in host defense and immune homeostasis. Infect. Dis. Rep. 2, pii: e9.

Kang, S.J., Tak, J.H., Cho, J.H., Lee, H.J., and Jung, Y.J. (2010). Stimulation of the endosomal TLR pathway enhances autophagy-induced cell death in radiotherapy of breast cancer Genes Genomics. 32, 599-606.

Kawai, T., and Akira, S. (2007). TLR signaling. Semin. Immunol. 19, 24-32.

Koons, A., Crandall, M., An, G.C., Shapiro, M.B., Kramer, S., and West, M.A. (2008). Even ephemeral endotoxin exposure establishes endotoxin tolerance. J. Trauma 64, 938-942.

Labonte, A.C., Tosello-Trampont, A.C., and Hahn, Y.S. (2014). The role of macrophage polarization in infectious and inflammatory diseases. Mol. Cells 37, 275-285.

Lang, T., and Mansell, A. (2007). The negative regulation of Toll-like receptor and associated pathways. Immunol. Cell Biol. 85, 425434.

Lee, J.Y., and Hwang, D.H. (2006). The modulation of inflammatory gene expression by lipids: Mediation through toll-like receptors. Mol. Cells 21, 174-185.

Liu, Z.J., Liu, X.L., Zhao, J., Shi, Y.J., Yan, L.N., Chen, X.F., Li, X.H., You, H.B., Xu, F.L., and Gong, J.P. (2008a). The effects of SOCS-1 on liver endotoxin tolerance development induced by a low dose of lipopolysaccharide are related to dampen NFkappaB-mediated pathway. Dig. Liver Dis. 40, 568-577.

Liu, Z.J., Yan, L.N., Li, X.H., Xu, F.L., Chen, X.F., You, H.B., and Gong, J.P. (2008b). Up-regulation of IRAK-M is essential for endotoxin tolerance induced by a low dose of lipopolysaccharide in Kupffer cells. J. Surg. Res. 150, 34-39.

Nakagawa, R., Naka, T., Tsutsui, H., Fujimoto, M., Kimura, A., Abe, T., Seki, E., Sato, S., Takeuchi, O., Takeda, K., et al. (2002). SOCS-1 participates in negative regulation of LPS responses. Immunity 17, 677-687.

Pluddemann, A., Mukhopadhyay, S., and Gordon, S. (2011). Innate immunity to intracellular pathogens: macrophage receptors and responses to microbial entry. Immunol. Rev. 240, 11-24.

Shuto, T., Kato, K., Mori, Y., Viriyakosol, S., Oba, M., Furuta, T., Okiyoneda, T., Arima, H., Suico, M.A., and Kai, H. (2005). Membrane-anchored CD14 is required for LPS-induced TLR4 endocytosis in TLR4/MD-2/CD14 overexpressing CHO cells. Biochem. Biophys. Res. Commun. 338, 1402-1409.

Xiong, Y., and Medvedev, A.E. (2011). Induction of endotoxin tolerance in vivo inhibits activation of IRAK4 and increases negative regulators IRAK-M, SHIP-1, and A20. J. Leukoc. Biol. 90, 1141-1148.

Xiong, Y., Qiu, F., Piao, W., Song, C., Wahl, L.M., and Medvedev, A.E. (2011). Endotoxin tolerance impairs IL-1 receptor-associated kinase (IRAK) 4 and TGF-beta-activated kinase 1 activation, K63linked polyubiquitination and assembly of IRAK1, TNF receptorassociated factor 6, and lkappaB kinase gamma and increases A20 expression. J. Biol. Chem. 286, 7905-7916.

Zacharioudaki, V., Androulidaki, A., Arranz, A., Vrentzos, G. Margioris, A.N., and Tsatsanis, C. (2009). Adiponectin promotes endotoxin tolerance in macrophages by inducing IRAK-M expression. J. Immunol. 182, 6444-6451. 